\title{
MEDICIÓN DEL RENDIMIENTO DE LA ORGANIZACIÓN COMO SISTEMA DE CONTROL DE GESTIÓN DEL CONTADOR
}

\author{
MEASUREMENT PERFORMANCE OF THE ORGANIZATION AS CONTROL \\ SYSTEM MANAGEMENT ACCOUNTANT
}

Román Arturo Valdivia Ramos*

Docente Asociado de la Facultad de Ciencias Contables - UNMSM

[Recepción: Setiembre de 2011/ Conformidad: Octubre de 2011]

\section{RESUMEN}

Ante el entorno de la Nueva Economía las transacciones y las medidas contables tradicionales para contabilizar éstas, por si mismas, no son suficientes para representar el valor de las empresas bajo las condiciones económicas actuales. Muchas veces se genera valor aún y cuando no haya ocurrido siquiera alguna de las transacciones usuales que se contabilizan en los negocios, bastan a veces expectativas de acciones futuras para que el valor de mercado de los negocios se vea afectado. Por ende, cada vez más empresas experimentan un diferencial entre los resultados obtenidos utilizando el enfoque de creación de valor y los obtenidos con el enfoque contable de rentabilidad y generación de utilidades, a través de un sistema de medición de rendimiento.

Tratamos de medir el rendimiento de una empresa, gerencia o área a través de medidas no - financieras, que nos den un indicativo (indicador) del comportamiento (desempeño) de una unidad orgánica, en un periodo determinado. Desde luego que este modelo o método de medición no es único, existen diversos y variados, lo importante es saber escoger el adecuado a los requerimientos y necesidades de la empresa o institución.

Estamos convencidos que los servicios (o intangibles), son lo más importante en toda empresa u organización y por consiguiente, debe ser medidos para después ser contabilizados. He ahí el reto que deben afrontar los contadores en un futuro cercano.

Palabras Clave: Medición, escala de valoración, factor limitante, plan de mejoramiento, Desempeño, Gestión.

\begin{abstract}
Given the environment of the New Economy transactions and traditional accounting measures to account for these, by themselves, are not sufficient to represent the value of companies under current economic conditions. Often creates value even if it did not happen even any unusual transactions that are posted in business, sometimes enough expectations of future actions for the market value of the business is affected. Thus, more and more companies experience a difference between the results obtained using the approach of creating value and those obtained with the accounting approach of profitability and revenue generation, through a performance measurement system.

We try to measure the performance of a company, or area management through measures not - financial, give us an indication (indicator) of the behavior (performance) of an organic unity in a given period. Of course, this model or method of measurement is not unique, there are many and varied, the important thing is to choose the appropriate to the requirements and needs of the company or institution.

We believe that the services (or intangibles) are the most important thing in any company or organization and therefore must be measured and then be counted. This is the challenge faced by accountants in the near future
\end{abstract}

Keywords: Measurement, assessment scale, limiting factor, improvement plan, Performance Management.

* Docente Asociado de la Facultad de Ciencias Contables- UNMSM, Licenciado en Administración - UNMSM. E-mail: arturo.valdivia.ramos@gmail.com 


\section{INTRODUCCIÓN}

En el presente artículo, señalaremos una serie de medidas que le van a permitir al contador, medir el rendimiento o performance contable de la gerencia, área o unidad que tenga a su cargo; es decir saber si su unidad funciona eficientemente y cuales son sus factores limitantes.

No debemos olvidar la frase de Peter F. Drucker: "No se puede dirigir lo que no se mide" y eso, es lo que pretendemos explicar. Claro está, que no es fácil medir intangibles como son los servicios (en este caso contables), difícil es contabilizarlos.

Debemos aprender a vivir con las siguientes frases:

1. "No se puede dirigir lo que no se mide"

2. "Lo único permanente es el cambio".

Debemos empezar a preocuparnos por medir los inmateriales que como sabemos, representan el activo más importante de toda empresa $\mathrm{u}$ organización. Los contadores contabilizan tangibles: registran materias primas, productos, inmuebles, maquinarias, equipos, dinero, en fin todo aquello que es susceptible de medir, pesar, valorar en términos monetarios (dinero) o crematísticos. Y de hecho, la mejor forma de medir es a través del valor económico. En esto, no hay ninguna duda

¿Pero ... acaso el ambiente, los stakeholders, el liderazgo, el entorno, el mercado, el cliente, la estructura organizacional, el intelecto de los trabajadores, etc., no tienen un valor económico o mejor dicho un valor contable? La respuesta clara y directa es $\mathrm{Si}$. Se pueden medir, pero aún no se pueden contabilizar. Si empezamos a diseñar una Escala (o como quiera llamársele) de Valoración, normalizada, estandarizada, consensuada y que sea aceptada y entendida por todos para medir intangibles, ya estaremos dando los pasos para alcanzar el futuro. Entonces, debemos crear o diseñar una Escala de Valoración Convencional de uso común para las empresas.

El problema que enfrentamos, es que no existe un método o Escala de Valoración que sea aceptado por todos. Pero, esto no significa que haya habido progreso, lo que sucede es que el mundo empresarial es plural, abierto e interdisciplinario, por lo tanto, la Medición del Rendimiento requiere de la participación no solo de contadores sino también de administradores, economistas, ingenieros e incluso de abogados, para formular de la mejor forma posible los datos, para luego convertirlos en información y usarlos para la toma de decisiones. Pero, es el contador el responsable de la performance.

También debe estar claro que una sola Medición del Rendimiento no es suficiente, se requiere diversas medidas. El asunto clave es saber cuáles son.

Samuel A. Mantilla, por ejemplo, señala las siguientes:
a. Las 4 perspectivas de Kaplan y Norton
b. Las 6 dimensiones de Fitzgerald.
c. Medición del capital intelectual.
d. Medidas de mejoramiento de procesos.
e. Desempeño basado en valor.
f. Mediciones No - Financieras.
g. Tipos de información.
h. Excelencia de negocios.
i. Prisma del desempeño, entre otros.

Pero también, existen algunos defectos comunes en la Medición del Rendimiento o Desempeño, siendo los más comunes:

a. Exceso de medición.

La abundante cantidad de mediciones no garantiza que se esté midiendo bien. Es indispensable usar las mediciones claves. 
b. Medición táctica o procedimental. No sirve para dirigir la organización.

c. Medidas inapropiadas

Si la medición no es la apropiada, nos puede llevar a errores.

d. Falla en actuar sobre los datos medidos La medición debe guiar la conducta, es decir, nos orienta a la mejor toma de decisiones.

e. Medidas hacia atrás, centradas en lo histórico Su utilidad es muy limitada. Solo sirve para hacer tendencias.

f. Entendimiento equivocado de lo que debe ser medido críticamente.

Más importante que la medida es que se va a medir. Una falla en este entendimiento es fatal. No se trata de medir por medir, sino medir lo que se necesita medir.

Por otro lado, una buena medición debe tener algunas características y estas son:

a. Confiable para el propósito.

b. Con una muestra adecuada.

c. Cuantificadora de la incertidumbre

d. Con rastreabilidad definida.

e. No perturbe al sistema que se mide.

f. Resumida adecuadamente.

g. Oportuna.

h. Exacta.

i. Comprensible.

j. Integrada.

Sin embargo, insistimos, no existe una fórmula mágica que pueda resolver todos los problemas de Medición del Rendimiento, nos enfrentamos a problemas comunes, como son:

a. No señalar la causa y el efecto.

b. Reforzar los aislamientos organizacionales.

c. Imagen borrosa de los problemas clave. d. Datos no - confiables o inexactos.

e. Consumo excesivo de tiempo y costos.

f. Filtración de los resultados.

g. Sin conexiones con la gestión del desempeño.

Por lo tanto, los elementos clave (no financieros), para hacer una buena Medición del Rendimiento, serán:
a. La estructura organizacional actual.
b. La auto - evaluación
c. Los procesos
d. El Benchmarking
e. El cliente
f. La calidad
g. El trabajador
h. Las buenas prácticas
i. La excelencia
j. El medio ambiente.

$\mathrm{Y}$ es que, los indicadores no - financieros cada vez tienen mayor importancia (siempre fueron importantes; tienen y tendrán más valor económico que los tangibles o materiales ahora y en el futuro) en la contabilidad. Lo que sucede es que aún no pueden contabilizarse, pero si medirse.

Esta medición, se traduce en:
a. Porcentaje
b. Peso
c. Volumen
d. Número
e. Tendencia
f. Gráfica, cuadro o tabla, etc., que debe mos convertirlo en dinero, para luego contabilizarlo.

Una "ayuda" que facilitará el empleo de indicadores para la Medición del Rendimiento, es la siguiente:

a. Defina lo que desea medir (toda la orga nización o parte de ella).

b. Divida en áreas, partes o secciones lo escogido.

c. Elabore y/o diseñe una Escala de Valoración. 
d. Aplique fórmulas, cuadros y gráficos.

e. Modifique lo hecho o actuado (mejora continua)

Lo que si está claro, es que si se quiere que los indicadores cumplan realmente con su objetivo, deben cumplir lo siguiente:

a. Cuantificables

Deben arrojar resultados cuantitativos.

b. Alineación

Uno es consecuencia de otro. Debe haber una relación causa - efecto.

c. Confiabilidad.

Estar libres de errores.

d. Eficiencia

El beneficio debe ser mayor que su costo.

e. Mejoramiento continuo

Orientarse al mejoramiento perpetuo.

f. Eficacia

Dar exactamente en el objetivo que se persigue ("dar en el bull o blanco")

(Tomado de Capital Intelectual \&

Contabilidad del Conocimiento de

Samuel Alberto Mantilla B. y adaptado por este autor).

Todas las empresas de una u otra forma fabrican productos $\mathrm{y} / \mathrm{o}$ brindan servicios a través de procesos de ingresos y salidas de insumos ó información. Estos procesos no solo son dependientes entre sí, sino que tienen una relación de dependencia con la empresa. "En toda empresa, debe existir al menos una limitación, de no ser así, sus beneficios serían infinitos".

Para mejorar los productos y/o servicios, entonces, se debe gestionar de la mejor forma posible las limitaciones que existen en dicha organización.
Si la capacidad de una empresa para generar valor está limitada, bien por el mercado, o por no tener capacidad suficiente, entonces la identificación de las limitaciones, será el primer paso que tendremos que dar para determinar en qué sub - factores se deben enfocar los esfuerzos de mejora. Este es el Primer Paso (Identificación de la(s) limitación(es))

El Segundo Paso (decidir como explotar la(s) limitación(es)), consiste en gestionar eficientemente dichas limitaciones para conseguir el máximo valor. Si la limitación puede ser superada sin llevar a cabo grandes inversiones, debemos hacerlo y luego volver de nuevo al Primer Paso. Si por el contrario, la limitación es un recurso productivo costoso (una máquina, por ejemplo), habrá que asegurar que dicho recurso esté trabajando todo el tiempo posible, dado que cada minuto que no trabaje parará todo el sistema, como señala José Luis Iglesias Sánchez, catedrático de la Universidad de Vigo / España.

El Tercer Paso, consiste en subordinar todas las acciones y políticas a la decisión anterior. También hay que tener en cuenta que la subordinación supone, como ocurre en las empresas de servicios, (como es el caso que estamos estudiando y analizando), que los recursos No limitados (ascensores, recepción, limpieza, aseo, etc.), deben tener capacidad sobrante para dar apoyo a los recursos limitados, lo que significa que si la limitación de una División viene por inventarios o costos, para evitar perder clientes en el futuro, los otros servicios o divisiones de la Gerencia de Contabilidad y Finanzas, han de tener la capacidad suficiente para evitar molestias a los clientes por demoras en los servicios, lo que llevaría a una pérdida de ingresos futuros. La capacidad de los recursos No limitados ha de ser, por consiguiente, superior a la estructura necesaria para su funcionamiento, dado que han de proteger el funcionamiento de los recursos limitados. 
La Teoría de las Limitaciones a través de este paso nos enseña que se debe equilibrar el flujo productivo y no la capacidad, lo que significa que solamente los recursos limitados no tendrán capacidad de protección (toda su capacidad debe ser utilizada) en cambio, los recursos No limitados, han de ser capaces de producir lo que necesita la limitación y además protegerlo de cualquier perturbación.

También la subordinación significa que las políticas empresariales (como los incentivos, bonificación por rendimiento, etc.), han de subordinarse a la limitación.

Cuando se ha desarrollado adecuadamente el Tercer Paso (subordinación), deberá darse el Cuarto Paso.

Este consiste, en llevar a cabo las inversiones operativas necesarias para poder satisfacer una mayor demanda (cuando la limitación es física) o en cambiar los sistemas ó políticas (cuando la limitación es de servicios). Cuando se da este Cuarto Paso y se ha roto la limitación, habrá que volver a empezar con el Primer Paso, dado que, de nuevo surgirá en otra parte de la organización, otra limitación. Este proceso se conoce como "proceso de mejora continua".

Como se ha indicado, el factor limitante no es nada fácil, se requiere de técnica especializada, matemática y estadística.

La Política Financiera puede ser la de maximizar el Capital de Trabajo o bien la de mantenerlo en un nivel adecuado. Este último es más conveniente si es que la empresa necesita utilizar créditos pasivos para aumentar su capacidad de rotación.

La inspección continua o permanente a este indicador de Capital de trabajo, ayuda a mantener el equilibrio de la Liquidez de las Cuentas por Pagar.

\section{CONCLUSIONES}

1. El análisis de las variaciones, representa un punto esencial para la evaluación del desempeño de los gerentes.

2. La variación en los resultados debe ser siempre objeto de análisis, enfatizándose en los factores causales claves que incidieron en la utilidad o pérdida del negocio, esto permitirá evaluar adecuadamente el desempeño del gerente.

3. Se pudo observar como las variaciones favorables no implicaban necesariamente un desempeño favorable; en forma similar ocurría con las variaciones desfavorables que no se traducían en desempeños desfavorables, sino que la evaluación del desempeño está en función del análisis resultante de la interrelación de los factores que originan las variaciones sin perder de vista el contexto estratégico del negocio que se esté evaluando.

4. Los cambios ocurridos en el entorno empresarial han demandado nuevas técnicas para gestionar la producción y los servicios, esto hace que los análisis de los resultados estén acorde con la evolución de los sistemas de gestión, en tal sentido, el análisis de los resultados será muy limitado si no se toma en consideración el contexto estratégico del negocio.

5. La Contabilidad es el lenguaje de los negocios y sabe hacia donde debe ir, es decir, a la medición y contabilización de los intangibles. Si el negocio, la organización, la administración, la gestión, la economía de la empresa cambian; la contabilidad, la auditoría, el costeo, deben cambiar también.

Vol. 19(36) 2011 | QUIPURAMAYOC / 19 
6. Los problemas contables ahora giran alrededor de la medición. Un nuevo sistema de mediciones será el fundamento de la Contabilidad del mañana.

\section{REFERENCIAS BIBLIOGRÁFICAS}

1. Montaño, G.A. (1975). Diagnóstico Industrial / Controles gráficos de dirección. Editorial Trillas, México. Primera reimpresión.

2. Mantilla, B.S. (2004). Capital Intelectual \& Contabilidad del Conocimiento. Ecoe Ediciones, Colombia. Tercera Edición.
3. Franklin, F.E. (2007). Auditoría Administrativa / Gestión estratégica del cambio. Editorial Pearson, México. Segunda edición.

4. Valdivia, R.A.(2011). Ingeniería de la Estructura de la Empresa. Libro en prensa, de próxima aparición.

5. Valdivia, R.A.(2011). Exposición sobre "Medición del Rendimiento de los Servicios", a los asesores de la Comisión de Reestructuración administrativa, orgánica y funcional de la UNMSM, Perú.

6. AECA No. 23 "Principios de Contabilidad de Gestión: Gerencia Estratégica de Costos”. 2001 